\title{
An Oscillation Criterion in Delay Differential Equations
}

\author{
George E. Chatzarakis \\ Department of Electrical and Electronic Engineering Educators, \\ School of Pedagogical and Technological Education (ASPETE) \\ 14121, N. Heraklio, Athens, Greece \\ geaxatz@otenet.gr, gea.xatz@aspete.gr
}

Abstract. Consider the first order linear delay differential equation

$$
x^{\prime}(t)+p(t) x(\tau(t))=0, \quad t \geq t_{0},
$$

where $p$ is a continuous function of nonnegative real numbers and the argument $\tau(t)$ is not necessarily monotone. Based on an iterative technique, a new oscillation criterion is established when the well-known oscillation conditions

$$
\limsup _{t \rightarrow \infty} \int_{\tau(t)}^{t} p(s) d s>1 \text { and } \quad \liminf _{t \rightarrow \infty} \int_{\tau(t)}^{t} p(s) d s>\frac{1}{e}
$$

are not satisfied. An example, numerically solved in MATLAB, is also given to illustrates the applicability and strength of the obtained condition over known ones.

Keywords: differential equation, non-monotone argument, oscillatory solution, nonoscillatory solution, Grönwall inequality.

2010 MSC No:34K06, 34K11.

\section{Introduction}

Consider the first-order delay differential equation

$$
x^{\prime}(t)+p(t) x(\tau(t))=0, \quad t \geq t_{0},
$$

where $p$ and $\tau$ are continuous on $\left[t_{0}, \infty\right), p(t) \geq 0, \tau(t)<t$ for $t \geq t_{0}$ and $\lim _{t \rightarrow \infty} \tau(t)=\infty$.

Definition 1.1. By a solution of $E)$ we mean a function which is continuous on $\left[\bar{t}_{*}, \infty\right)$ for some $t_{*} \geq t_{0}$, where $\bar{t}_{*}=\inf \left\{\tau(t): t \geq t_{*}\right\}$.

Definition 1.2. A solution of $(E)$ is say to be oscillatory, if it is has arbitrarily large zeros. Otherwise, it is called nonoscillatory. An equation is oscillatory if all its solutions oscillate.

In 1972, Ladas, Lakshmikantham and Papadakis [9], and in 1982, Koplatadze and Chanturija [8] proved that, if

$$
\limsup _{t \rightarrow \infty} \int_{\tau(t)}^{t} p(s) d s>1 \quad \text { or } \quad \alpha:=\liminf _{t \rightarrow \infty} \int_{\tau(t)}^{t} p(s) d s>\frac{1}{e}
$$

then all solutions of $(\mathrm{E})$ are oscillatory. 
Assume that the argument $\tau(t)$ is not necessarily monotone. Set

$$
h(t):=\sup _{s \leq t} \tau(s), \quad t \geq t_{0} .
$$

Clearly, the function $h(t)$ is nondecreasing and $\tau(t) \leq h(t)<t$ for all $t \geq t_{0}$.

In 2011 Braverman and Karpuz [1] proved that, if

$$
\limsup _{t \rightarrow \infty} \int_{h(t)}^{t} p(s) \exp \left(\int_{\tau(s)}^{h(t)} p(u) d u\right) d s>1
$$

then all solutions of (E) oscillate.

Several improvements were made to the above condition, see [2-5] to arrive at the recent form [5]

$$
\limsup _{t \rightarrow \infty} \int_{h(t)}^{t} p(s) \exp \left(\int_{\tau(s)}^{h(t)} p(u) \exp \left(\int_{\tau(u)}^{u} d_{\ell}(\xi) d \xi\right) d u\right) d s>1
$$

where

with

$$
d_{\ell}(t)=p(t)\left[1+\int_{\tau(t)}^{t} p(s) \exp \left(\int_{\tau(s)}^{t} p(u) \exp \left(\int_{\tau(u)}^{u} d_{\ell-1}(\xi) d \xi\right) d u\right) d s\right]
$$

$$
d_{0}(t)=p(t)\left[1+\int_{\tau(t)}^{t} p(s) \exp \left(\int_{\tau(s)}^{t} p(\omega) \exp \left(\lambda_{0} \int_{\tau(\omega)}^{\omega} p(u) d u\right) d \omega\right) d s\right]
$$

and $\lambda_{0}$ is the smaller root of the transcendental equation $\lambda=e^{\alpha \lambda}$.

In the present paper we establish a new iterative sufficient condition, involving lim sup, for the oscillation of all solutions of (E) when the argument is not necessarily monotone. The result obtained essentially improves known results in the literature.

\section{Main Results}

The proof of our main result is essentially based on the following lemmas.

Lemma 2.1. [6, Lemma 2.1.1] Assume that $h(t)$ is defined by (1.2). Then

$$
\alpha:=\liminf _{t \rightarrow \infty} \int_{\tau(t)}^{t} p(s) d s=\liminf _{t \rightarrow \infty} \int_{h(t)}^{t} p(s) d s .
$$

Lemma 2.2. [10] Assume that $h(t)$ is defined by (1.2), $\alpha \in(0,1 / e]$ and $x(t)$ is an eventually positive solution of (E). Then

$$
\liminf _{t \rightarrow \infty} \frac{x(h(t))}{x(t)} \geq \lambda_{0}
$$

where $\lambda_{0}$ is the smaller root of the transcendental equation $\lambda=e^{\alpha \lambda}$.

Theorem 2.3. Assume that $h(t)$ is defined by (1.2) and for some $\ell \in \mathbb{N}$

$$
\limsup _{t \rightarrow \infty} \int_{h(t)}^{t} p(s) \exp \left(\int_{\tau(s)}^{h(t)} p(u) \exp \left(\int_{\tau(u)}^{u} b_{\ell}(\xi) d \xi\right) d u\right) d s>1,
$$

where

with

$$
b_{\ell}(t)=p(t)\left[1+\int_{\tau(t)}^{t} p(s) \exp \left(\int_{\tau(s)}^{t} p(u) \exp \left(\int_{\tau(u)}^{u} b_{\ell-1}(\xi) d \xi\right) d u\right) d s\right] \text {, }
$$

$$
b_{0}(t)=p(t)\left[1+\int_{\tau(t)}^{t} p(s) \exp \left(\int_{\tau(s)}^{t} p(y) \exp \left(\int_{\tau(y)}^{y} p(\omega) \exp \left(\lambda_{0} \int_{\tau(\omega)}^{\omega} p(u) d u\right) d \omega\right) d y\right) d s\right]
$$

and $\lambda_{0}$ is the smaller root of the transcendental equation $\lambda=e^{\alpha \lambda}$. Then all solutions of (E) are oscillatory. 
Proof. Assume, for the sake of contradiction, that there exists a nonoscillatory solution $x(t)$ of $(\mathrm{E})$. Since $-x(t)$ is also a solution of $(\mathrm{E})$, we can confine our discussion only to the case where the solution $x(t)$ is eventually positive. Then there exists a $t_{1}>t_{0}$ such that $x(t)$ and $x(\tau(t))>0$ for all $t \geq t_{1}$. Thus, from (E) we have $x^{\prime}(t)=-p(t) x(\tau(t)) \leq 0$ for all $t \geq t_{1}$, which means that $x(t)$ is an eventually nonincreasing function of positive numbers. Now we divide (E) by $x(t)>0$ and integrate on $[\tau(t), t]$, so

$$
\int_{\tau(t)}^{t} \frac{x^{\prime}(u)}{x(u)} d u=-\int_{\tau(t)}^{t} p(u) \frac{x(\tau(u))}{x(u)} d u,
$$

or

$$
x(\tau(t))=x(t) \exp \left(\int_{\tau(t)}^{t} p(u) \frac{x(\tau(u))}{x(u)} d u\right) .
$$

Combining (E) and (2.5), we have

$$
x^{\prime}(t)+p(t) x(t) \exp \left(\int_{\tau(t)}^{t} p(u) \frac{x(\tau(u))}{x(u)} d u\right)=0 .
$$

We divide $(2.4)$ by $x(t)>0$ and integrate on $[\tau(t), t]$, so

$$
\int_{\tau(t)}^{t} \frac{x^{\prime}(\omega)}{x(\omega)} d \omega=-\int_{\tau(t)}^{t} p(\omega) \exp \left(\int_{\tau(\omega)}^{\omega} p(u) \frac{x(\tau(u))}{x(u)} d u\right) d \omega
$$

or

$$
x(\tau(t))=x(t) \exp \left(\int_{\tau(t)}^{t} p(\omega) \exp \left(\int_{\tau(\omega)}^{\omega} p(u) \frac{x(\tau(u))}{x(u)} d u\right) d \omega\right) .
$$

Combining (E) and (2.5), we have

$$
x^{\prime}(t)+p(t) x(t) \exp \left(\int_{\tau(t)}^{t} p(\omega) \exp \left(\int_{\tau(\omega)}^{\omega} p(u) \frac{x(\tau(u))}{x(u)} d u\right) d \omega\right)=0 .
$$

We divide (2.6) by $x(t)>0$ and integrate on $[\tau(s), t]$, so

$$
\int_{\tau(s)}^{t} \frac{x^{\prime}(y)}{x(y)} d y=-\int_{\tau(s)}^{t} p(y) \exp \left(\int_{\tau(y)}^{y} p(\omega) \exp \left(\int_{\tau(\omega)}^{\omega} p(u) \frac{x(\tau(u))}{x(u)} d u\right) d \omega\right) d y
$$

or

$$
x(\tau(s))=x(t) \exp \left(\int_{\tau(s)}^{t} p(y) \exp \left(\int_{\tau(y)}^{y} p(\omega) \exp \left(\int_{\tau(\omega)}^{\omega} p(u) \frac{x(\tau(u))}{x(u)} d u\right) d \omega\right) d y\right) .
$$

Integrating (E) from $\tau(t)$ to $t$, using (2.7), multiplying by $p(t)$ and taking into account the fact that $x^{\prime}(t)=$ $-p(t) x(\tau(t))$, we obtain

$$
\begin{aligned}
& x^{\prime}(t)+p(t) x(t) \\
& +p(t) x(t) \int_{\tau(t)}^{t} p(s) \exp \left(\int_{\tau(s)}^{t} p(y) \exp \left(\int_{\tau(y)}^{y} p(\omega) \exp \left(\int_{\tau(\omega)}^{\omega} p(u) \frac{x(\tau(u))}{x(u)} d u\right) d \omega\right) d y\right) d s=0 .
\end{aligned}
$$

Since $\tau(u) \leq h(u)$, clearly

$$
\begin{aligned}
& x^{\prime}(t)+p(t) x(t) \\
& +p(t) x(t) \int_{\tau(t)}^{t} p(s) \exp \left(\int_{\tau(s)}^{t} p(y) \exp \left(\int_{\tau(y)}^{y} p(\omega) \exp \left(\int_{\tau(\omega)}^{\omega} p(u) \frac{x(h(u))}{x(u)} d u\right) d \omega\right) d y\right) d s \leq 0 .
\end{aligned}
$$


Taking into account the fact that Lemmas 1 and 2 are satisfied, the last inequality becomes $x^{\prime}(t)$

$$
+p(t)\left[1+\int_{\tau(t)}^{t} p(s) \exp \left(\int_{\tau(s)}^{t} p(y) \exp \left(\int_{\tau(y)}^{y} p(\omega) \exp \left(\left(\lambda_{0}-\epsilon\right) \int_{\tau(\omega)}^{\omega} p(u) d u\right) d \omega\right) d y\right) d s\right] x(t) \leq 0
$$

or

$$
x^{\prime}(t)+b_{0}(t, \epsilon) x(t) \leq 0
$$

with

$$
b_{0}(t, \epsilon)=p(t)\left[1+\int_{\tau(t)}^{t} p(s) \exp \left(\int_{\tau(s)}^{t} p(y) \exp \left(\int_{\tau(y)}^{y} p(\omega) \exp \left(\left(\lambda_{0}-\epsilon\right) \int_{\tau(\omega)}^{\omega} p(u) d u\right) d \omega\right) d y\right) d s\right] .
$$

Applying the Grönwall inequality in (2.8), we obtain

$$
x(\tau(u)) \geq x(u) \exp \left(\int_{\tau(u)}^{u} b_{0}(\xi, \epsilon) d \xi\right) .
$$

Now we divide $(\mathrm{E})$ by $x(t)>0$, integrate on $[\tau(s), t]$ and use $(2.9)$, so

$$
-\int_{\tau(s)}^{t} \frac{x^{\prime}(u)}{x(u)} d u=\int_{\tau(s)}^{t} p(u) \frac{x(\tau(u))}{x(u)} d u \geq \int_{s}^{t} p(u) \exp \left(\int_{\tau(u)}^{u} b_{0}(\xi, \epsilon) d \xi\right) d u
$$

or

$$
x(\tau(s)) \geq x(t) \exp \left(\int_{\tau(s)}^{t} p(u) \exp \left(\int_{\tau(u)}^{u} b_{0}(\xi, \epsilon) d \xi\right) d u\right) .
$$

Integrating (E) from $\tau(t)$ to $t$ and using (2.10), we obtain

$$
x(t)-x(\tau(t))+x(t) \int_{\tau(t)}^{t} p(s) \exp \left(\int_{\tau(s)}^{t} p(u) \exp \left(\int_{\tau(u)}^{u} b_{0}(\xi, \epsilon) d \xi\right) d u\right) d s \leq 0 .
$$

Multiplying the last inequality by $p(t)$, we find

$$
p(t) x(t)-p(t) x(\tau(t))+p(t) x(t) \int_{\tau(t)}^{t} p(s) \exp \left(\int_{\tau(s)}^{t} p(u) \exp \left(\int_{\tau(u)}^{u} b_{0}(\xi, \epsilon) d \xi\right) d u\right) d s \leq 0
$$

which, in view of (E), becomes

$$
x^{\prime}(t)+p(t) x(t)+p(t) x(t) \int_{\tau(t)}^{t} p(s) \exp \left(\int_{\tau(s)}^{t} p(u) \exp \left(\int_{\tau(u)}^{u} b_{0}(\xi, \epsilon) d \xi\right) d u\right) d s \leq 0 .
$$

Hence, for sufficiently large $t$

$$
x^{\prime}(t)+p(t)\left[1+\int_{\tau(t)}^{t} p(s) \exp \left(\int_{\tau(s)}^{t} p(u) \exp \left(\int_{\tau(u)}^{u} b_{0}(\xi, \epsilon) d \xi\right) d u\right) d s\right] x(t) \leq 0
$$

or

$$
x^{\prime}(t)+b_{1}(t, \epsilon) x(t) \leq 0
$$

where

$$
b_{1}(t, \epsilon)=p(t)\left[1+\int_{\tau(t)}^{t} p(s) \exp \left(\int_{\tau(s)}^{t} p(u) \exp \left(\int_{\tau(u)}^{u} b_{0}(\xi, \epsilon) d \xi\right) d u\right) d s\right] .
$$

Following the above procedure, we can inductively construct the inequalities

$$
x^{\prime}(t)+b_{\ell}(t) x(t, \epsilon) \leq 0, \quad(\ell \in \mathbb{N}),
$$


where

$$
b_{\ell}(t, \epsilon)=p(t)\left[1+\int_{\tau(t)}^{t} p(s) \exp \left(\int_{\tau(s)}^{t} p(u) \exp \left(\int_{\tau(u)}^{u} b_{\ell-1}(\xi, \epsilon) d \xi\right) d u\right) d s\right]
$$

and

$$
x(\tau(s)) \geq x(h(t)) \exp \left(\int_{\tau(s)}^{h(t)} p(u) \exp \left(\int_{\tau(u)}^{u} b_{\ell}(\xi, \epsilon) d \xi\right) d u\right) .
$$

Integrating (E) from $h(t)$ to $t$, using (2.11) and if we omit $x(t)>0$, we have

$$
x(h(t))\left[\int_{h(t)}^{t} p(s) \exp \left(\int_{\tau(s)}^{h(t)} p(u) \exp \left(\int_{\tau(u)}^{u} b_{\ell}(\xi, \epsilon) d \xi\right) d u\right) d s-1\right]<0,
$$

which means that

$$
\limsup _{t \rightarrow \infty} \int_{h(t)}^{t} p(s) \exp \left(\int_{\tau(s)}^{h(t)} p(u) \exp \left(\int_{\tau(u)}^{u} b_{\ell}(\xi, \epsilon) d \xi\right) d u\right) d s \leq 1 .
$$

Since $\epsilon$ may be taken arbitrarily small, this inequality contradicts (2.2).

The proof of the theorem is complete.

Example 2.4. Consider the delay differential equation

with

$$
x^{\prime}(t)+\frac{1}{8} x(\tau(t))=0, \quad t \geq 0,
$$

$$
\tau(t)= \begin{cases}-t+12 k-1, & \text { if } t \in[6 k, 6 k+1] \\ 6 k-2, & \text { if } t \in[6 k+1,6 k+2] \\ 3 t-12 k-8, & \text { if } t \in[6 k+2,6 k+3] \\ -0.5 t+9 k+2.5, & \text { if } t \in[6 k+3,6 k+5] \\ 5 t-24 k-25, & \text { if } t \in[6 k+5,6 k+6]\end{cases}
$$

where $k \in \mathbb{N}_{0}$ and $\mathbb{N}_{0}$ is the set of nonnegative integers. By (1.2), we see that

$$
h(t)=\left\{\begin{array}{ll}
6 k-1, & \text { if } t \in[6 k, 6 k+7 / 3] \\
3 t-12 k-8, & \text { if } t \in[6 k+7 / 3,6 k+3] \\
6 k+1, & \text { if } t \in 6 k+3,6 k+26 / 5] \\
5 t-24 k-25, & \text { if } t \in[6 k+26 / 5,6 k+6]
\end{array} .\right.
$$

It is easy to see that $\alpha=\liminf _{k \rightarrow \infty} \int_{6 k+5}^{6 k+6} \frac{1}{8} d s=0.125$ and $\lambda_{0} \simeq 1.15537$.

Observe that the function $F_{\ell}: \mathbb{R}_{0} \rightarrow \mathbb{R}_{+}$defined as

$$
F_{\ell}(t)=\int_{h(t)}^{t} p(s) \exp \left(\int_{\tau(s)}^{h(t)} p(u) \exp \left(\int_{\tau(u)}^{u} b_{\ell}(\xi) d \xi\right) d u\right) d s,
$$

attains its maximum at $t=6 k+26 / 5$, for every $\ell \in \mathbb{N}$. Specifically, by using an algorithm on MATLAB software, we obtain

$$
F_{1}(t=6 k+26 / 5)=\int_{6 k+1}^{6 k+26 / 5} p(s) \exp \left(\int_{\tau(s)}^{6 k+1} p(u) \exp \left(\int_{\tau(u)}^{u} b_{1}(\xi) d \xi\right) d u\right) d s \simeq 1.037
$$

and therefore, condition (2.2) of Theorem 1 is satisfied for $\ell=1$. Thus, all solutions of (2.12) are oscillatory. Observe, however, that

$$
\begin{gathered}
\limsup _{k \rightarrow \infty} \int_{6 k+1}^{6 k+26 / 5} \frac{1}{8} d s \simeq 0.525<1 \text { and } \alpha=0.125<\frac{1}{e} \\
\limsup _{t \rightarrow \infty} \int_{h(t)}^{t} p(s) \exp \left(\int_{\tau(s)}^{h(t)} p(u) d u\right) d s \simeq 0.6226<1
\end{gathered}
$$




$$
\limsup _{t \rightarrow \infty} \int_{h(t)}^{t} p(s) \exp \left(\int_{\tau(s)}^{h(t)} p(u) \exp \left(\int_{\tau(u)}^{u} d_{1}(\xi) d \xi\right) d u\right) d s \simeq 0.9269<1
$$

That is, none of the conditions (1.1), (1.3)and (1.4) (for $\ell=1$ ) is satisfied.

Comments. It is worth noting that the improvement of condition (2.2) to the corresponding condition (1.1) is significant, approximately $97.52 \%$, if we compare the values on the left-side of these conditions. Also, the improvement compared to conditions (1.3) and (1.4) is very satisfactory, around $66.56 \%$, and $11.88 \%$, respectively. In addition, observe that condition (1.4) does not lead to oscillation for first iteration. On the contrary, condition (2.2) is satisfied from the first iteration. This means that our condition is better and much faster than (1.4).

\section{References}

[1] E. Braverman, B. Karpuz, On oscillation of differential and difference equations with non-monotone delays, Appl. Math. Comput., 218 (2011) 3880-3887. https://doi.org/10.1016/j.amc.2011.09.035

[2] G. E. Chatzarakis, Differential equations with non-monotone arguments: Iterative Oscillation results, J. Math. Comput. Sci., 6(5) (2016), 953-964.

[3] G. E. Chatzarakis, On oscillation of differential equations with non-monotone deviating arguments, Mediterr. J. Math., 14(2) (2017), Art. 82, 17 pp. https://doi.org/10.1007/s00009-017-0883-0

[4] G. E. Chatzarakis and I. Jadlovská, Improved iterative oscillation tests for firs-order deviating differential equations, Opuscula Math., 38(3) (2018), 327 https://doi.org/10.7494/opmath.2018.38.3.327

[5] G. E. Chatzarakis and I. Jadlovská, Oscillations in differential equations caused by non-monotone arguments, 2018, to appear.

[6] L. H. Erbe, Qingkai Kong and B.G. Zhang, Oscillation Theory for Functional Differential Equations, Marcel Dekker, New York, 1995.

[7] N. Fukagai and T. Kusano, Oscillation theory of first order functional-differential equations with deviating arguments, Ann. Mat. Pura Appl. 136 (1984), 95- 117. https://doi.org/10.1007/bf01773379

[8] R. G. Koplatadze and T. A. Chanturija, Oscillating and monotone solutions of first-order differential equations with deviating argument, (Russian), Differentsial/nye Uravneniya 18 (1982), 1463-1465, 1472.

[9] G. Ladas, V. Lakshmikantham and L. S. Papadakis, Oscillations of higher-order retarded differential equations generated by the retarded arguments, Delay and Functional Differential Equations and their Applications, Academic Press, New York, 1972, 219-231. https://doi.org/10.1016/b978-0-12-627250-5.50013-7

[10] D. Zhou, On some problems on oscillation of functional differential equations of first order, J. Shandong University 25 (1990), 434-442. 